\title{
Strategic uncertainty, indeterminacy, and the formation of international environmental agreements
}

\author{
By Daiju Narita ${ }^{a}$ and Ulrich J. Wagner ${ }^{b}$ \\ a Graduate School of Economics and Business Administration, Hokkaido University, Kita 9 Nishi 7, \\ Kita-ku, Sapporo 060-0809, Japan, and Kiel Institute for the World Economy, Germany; \\ e-mail: daiju.narita@econ.hokudai.ac.jp \\ ${ }^{b}$ Department of Economics, University of Mannheim, Germany
}

\begin{abstract}
Since the end of the Kyoto Protocol, global climate negotiations have shifted away from setting binding short-run targets on emissions towards placing long-term limits on global warming. We investigate how this alters the incentives for participation in a technology-centred international environmental agreement (IEA) where countries choose between conventional abatement and a breakthrough abatement technology that exhibits a network externality. When switching technologies is costly, we obtain that equilibrium adoption is indeterminate because the future adoption rate is subject to strategic uncertainty. Participation in an IEA that mandates the adoption of the breakthrough technology will be complete only if countries expect that all other countries will adopt eventually. Long-run temperature targets can be regarded as a device to coordinate countries' expectations on that outcome.
\end{abstract}

JEL classifications: 054, 033, H87.

\section{Introduction}

In recent years, a global consensus has emerged that explicit long-term targets for the emissions of greenhouse gases (GHGs) are meaningful for promoting the mitigation of climate change. For example, both the Copenhagen Accord in 2009 and the Paris Agreement in 2015 make explicit reference to a $2^{\circ}$ long-term target for global warming. ${ }^{1}$ The European Union currently pursues the target of reducing GHG emissions by $80 \%$ to $95 \%$ by 2050 (compared to 1990 levels), and the group of G7 countries agreed to reduce emissions by $40 \%$ to $70 \%$ by 2050 (compared to 2010 levels). Targets such as these can help pollution abatement in the long-run by promoting large-scale investments in research and

1 In addition, the Paris Agreement also states the need for 'pursuing efforts to limit the temperature increase to $1.5^{\circ} \mathrm{C}$ above pre-industrial levels.' 
development of new technologies that will be deployed only in the (distant) future, such as nuclear fusion or carbon capture from the air.

Does the mere existence of a long-term target make countries more willing to engage in an international environmental agreement (IEA) in the near term? The answer to this question is not clear because compliance with the target cannot be taken for granted. What seems clear, however, is that meeting the long-term targets mentioned above will require a series of technological breakthroughs to achieve a decoupling of economic growth and carbon emissions. In that sense, the existence of a long-term target-for as ambitious or idealistic as it may seem-raises an expectation among the stakeholders that breakthroughs will eventually occur. This feature of targets begs the question of how expectations about cooperation in the future can influence the formation of IEAs in the present. The literature on self-enforcing treaties has highlighted the role of coordination when participation in an IEAs hinges upon a tipping point (Barrett, 2003, 2006) ${ }^{2}$ but little is known so far about how coordination can be achieved when treaty formation takes place over time.

This study shows that expectations about collective pollution abatement in the future can influence the formation of IEAs even in the near term. We model treaty formation as a dynamic game of technology choice where country behaviour is conditioned by a network externality associated with one of the abatement technologies, and by switching costs that are convex in the number of countries that switch technologies. The model gives rise to two types of equilibrium dynamics of technology adoption. In the first case, adoption follows a determinate path which leads to either full cooperation or no cooperation, depending on the initial state. In the second case, the dynamics are indeterminate, with stable paths leading to both full cooperation and no cooperation. The path chosen depends on countries' expectations. That is, the size of a self-enforcing treaty is driven to some extent by subjective beliefs that countries hold about their ability to coordinate policies. This case emerges when countries are patient, face a low switching cost, and when there are high scale effects of operational cost. In the case of climate change, such an indeterminacy in the adoption of abatement technologies provides a rationale for negotiating long-term abatement targets that align expectations of countries on mutually beneficial diffusion trajectories.

The paper is organized as follows. In Section 2, we set up a basic two-stage model with frontrunner and follower countries of a new abatement technology to illustrate how indeterminate dynamics could emerge. Next, we generalize the model by allowing countries to choose the timing of treaty adoption. We discuss both a two-period version of the model (in Section 3.1) and an infinite-horizon game (in Section 3.2), and derive the equilibrium dynamics in Section 3.3. Section 4 discusses the results of the model, and concluding remarks are given in Section 5.

\section{A model of technology adoption with frontrunner and follower countries}

We consider the formation of an IEA that regulates pollution abatement by multiple technological options. Here, we set the focus of the model discussions on the adoption of a technology that involves a network externality. Network externalities are known to create strategic complementarities that may lead to tipping points. Our model focuses on the

2 The concept of tipping points used in this context relates to the seminal work by Thomas Schelling (1978). For a more recent, informal treatment, see the book by Malcolm Gladwell (2000). 
diffusion process of an abatement technology which is not instantaneous but occurs over time. In combination with the network externality, the delay engenders strategic uncertainty about future adoption decisions, transforming coordination on the good outcome into a non-trivial problem. ${ }^{3}$

Network externalities have been alleged to play a role for various IEAs. For example, Barrett (2003, Chapter 9) discusses the equipment standards for oil tankers set by the International Convention for the Prevention of Pollution from Ships (MARPOL), which aim to reduce oil discharge to the sea at the time of exchange of ballast water and oil in the tank. With the equipment standards, tanker owners reap greater benefits from installing new equipment as more ports comply with the agreement, hence favouring higher participation in the treaty after the group of participating countries reaches a critical size. Another example is the development and production of substitute products for CFCs after the latter were regulated by the Montreal Protocol. It is conceivable that, once the market size of the substitutes became large, DuPont and others producers stood to gain more from producing the substitutes than from producing CFCs and thus preferred a treaty with universal participation (e.g., Parson, 2003; Sunstein, 2007). In the context of climate change, network externalities will likely arise in the deployment of hydrogen-based transportation systems, specifically when combining a new automobile technology and a supporting infrastructure of fuel supply. Moreover, in a network of carbon capture and storage (CCS) operations whose capture and storage sites are linked to each other by pipelines for carbon dioxide, the costs of accessing the CCS network facing an emitter are likely decreasing in the number of other emitters using the network.

For an illustration of the problem we are interested in, we start by discussing a simple case where countries are grouped into frontrunners and followers, and changing groups is not allowed. ${ }^{4}$ Our model setup extends Barrett's (2006) model of a treaty on technology adoption. Countries have a choice between conventional abatement $q$ and the adoption of 'breakthrough technology' labelled 'Technology X'. The latter has the distinctive features that it generates zero emissions and exhibits increasing returns to adoption. In Barrett's model, a one-shot game is played among $N$ countries, where:

$$
\begin{aligned}
\pi_{i} & =b_{x}\left(x_{i}+\sum_{j \neq i}^{N} x_{j}\right)-\frac{c_{x}}{N}\left(N-\sum_{j \neq i}^{N} x_{j}\right) x_{i} \\
& +b\left[\left(1-x_{i}\right) q_{i}+\sum_{j \neq i}^{N}\left(1-x_{j}\right) q_{j}\right]-c_{0}\left(1-x_{i}\right) \frac{q_{i}^{2}}{2}
\end{aligned}
$$

is the payoff function for country $i, x_{i}$ is the indicator of adoption of Technology $\mathrm{X}$ by country $i\left(x_{i} \in\{0,1\}\right), q_{i}$ is country $i$ 's abatement rate by using the conventional technology, and $b_{x}, c_{x}, b, c_{0}$ are strictly positive coefficients representing the marginal benefit of adopting Technology $\mathrm{X}$, the total cost of using Technology $\mathrm{X}$, the marginal benefit from conventional abatement and the marginal cost of conventional abatement, respectively. Barrett has shown that this one-shot game gives rise to a 'tipping treaty' as both universal adoption and non-adoption of Technology $\mathrm{X}$ are Nash equilibria. If the equilibria are

3 Strategic uncertainty arises not due to stochastic elements in the payoff functions but due to the uncertainty concerning the actions and beliefs of other players.

4 This assumption is relaxed in Section 3 below, where each country is allowed to switch between technologies at any time. 
ranked, successful coordination among the countries in favour of technology adoption increases the payoff for at least some of them.

Using functional forms similar to Barrett's, we now show that the addition of a temporal dimension limits the possibility of coordinated actions by countries and is conducive to a pattern of indeterminate adoption dynamics. The prospects for successful coordination are worse than in the one-shot game because some countries are unable to adopt the technology at a given moment in time.

We consider a game in two stages where each country belongs to one of two groups, technological frontrunners or followers. Decisions of technology choice by countries are made sequentially. In period 1 , group $1(i=1, \ldots, M$ where $M<N-1)$ countriestechnological frontrunners with the technical capacity to use Technology $\mathrm{X}$ from the beginning-make a decision about whether they introduce Technology X. Group 2 countries $(i=M+1, \ldots, N)$-followers-acquire the ability to introduce Technology X only in period 2. The assumption of sequential decisions is chosen for the sake of simplicity and to reflect two types of costs, namely: (i) a prohibitive cost of reverting from a new abatement technology to the conventional one; and (ii) a prohibitive cost facing some countries associated with the early adoption of a new technology. These assumptions will be relaxed in Section 3 below where we consider a simultaneous-moves game and reversible technology choices.

Group 1 countries (frontrunners) adopt Technology X in period 1 if the present value expected payoff for individual countries favours adoption. That is, when deciding on adoption or non-adoption, frontrunners seek to maximize their individual payoffs rather than joint payoffs. The present value expected payoff for countries $1, \ldots, M$ evaluated in period 1 is given by:

$$
\begin{aligned}
\Pi_{i}= & \pi_{i}^{t=1}\left(x_{11}, \ldots, x_{i 1}, \ldots, x_{M 1}\right)+\beta E\left[\pi_{i}^{t=2}\left(x_{12}, \ldots, x_{i 2}, \ldots, x_{N 2}\right)\right] \\
= & b_{x}\left(x_{i 1}+\sum_{i \neq i}^{M} x_{j 1}\right)-\frac{c_{x}}{N}\left(N-\sum_{i \neq i}^{M} x_{j 1}\right) x_{i 1} \\
& +b\left[\left(1-x_{i 1}\right) q_{i 1}+\sum_{i \neq i}^{M}\left(1-x_{j 1}\right) q_{j 1}\right] \\
& -c_{0}\left(1-x_{i 1}\right) \frac{q_{i 1}^{2}}{2}+\beta\left[b_{x}\left(x_{i 2}+\sum_{j \neq i}^{N} x_{j 2}\right)-\frac{c_{x}}{N}\left(N-\sum_{i \neq i}^{N} x_{j 2}\right) x_{i 2}\right] \\
& +\beta\left\{b\left[\left(1-x_{i 2}\right) q_{i 2}+\sum_{j \neq i}^{N}\left(1-x_{j 2}\right) q_{j 2}\right]-c\left(1-x_{i 2}\right) \frac{q_{i 2}^{2}}{2}\right\}
\end{aligned}
$$

where $x_{j t} \in\{0,1\}$ is the indicator for country $j$ 's adoption of Technology $\mathrm{X}$ in period $t \in\{1,2\}$, and $\beta \in(0,1)$ is the discount factor.

We now show that, in certain cases, rational decision-making by countries can lead to more than one possible outcome. Which one of them will be realized depends on what countries expect at present. Suppose that the adoption of Technology X in period 1 makes 
frontrunners better off only if Technology $\mathrm{X}$ is also adopted by followers in period 2. This is true if the following conditions are satisfied: ${ }^{5}$

$$
\begin{array}{r}
\left(b_{x}-\frac{b^{2}}{2 c_{0}}\right) M-\frac{c_{x}}{N}(N-M+1)+\frac{b^{2}}{2 c_{0}}+\beta\left[\left(b_{x}-\frac{b^{2}}{2 c_{0}}\right) N-\frac{c_{x}}{N}+\frac{b^{2}}{2 c_{0}}\right] \\
\left(b_{x}-\frac{b^{2}}{2 c_{0}}\right) M-\frac{c_{x}}{N}(N-M+1)+\frac{b^{2}}{2 c_{0}}+\beta\left[\left(b_{x}-\frac{b^{2}}{2 c_{0}}\right) M-\frac{c_{x}}{N}(N-M+1)+\frac{b^{2}}{2 c_{0}}\right]<0
\end{array}
$$

Figure 1 depicts the payoff schedules corresponding to this case. Non-adoption by all countries in both periods is one Nash equilibrium of the game, yet it is dominated by the other Nash equilibrium in which all countries adopt by the end of period 2. However, because the decision on technology adoption is taken sequentially, a coordination problem arises in both periods. For instance, even if frontrunners manage to coordinate on adoption in period 1 , coordination might fail in period 2 as followers might still choose non-adoption of Technology X. Conversely, conditions (3) and (4) imply that adoption of Technology X by frontrunners is not optimal if followers do not follow suit. And followers have no incentive to adopt Technology $\mathrm{X}$ if frontrunners have not adopted Technology X beforehand.

As the incentives of the two groups are interrelated in a circular fashion there is more than one possible outcome. The outcome could in fact be determined by frontrunners' expectations about future actions by followers. Success or failure of treaty coordination in period 2-and hence the rate of technology adoption in the future-is subject to strategic uncertainty in period 1 . That is, unless one places additional assumptions on the structure of expectations, frontrunners decide on technology adoption based on their beliefs about the future outcome. Despite the subjective nature of these beliefs, frontrunners determine the eventual adoption rate of Technology $\mathrm{X}$ by directly shaping the followers' incentive for technology adoption in period 2. As a consequence, the diffusion of Technology X could be driven by a subjective factor, and followers have little influence in shaping such subjective beliefs. Previous analyses have given little attention to this issue, as they have been based on a one-shot game of technology adoption where the problem of coordination boils down to a matter of successful political negotiations at one point in time. Such a framework rules out the intertemporal coordination problem we examine in this paper.

To be sure, the problem of multiplicity arising in the two-period model we consider here could be avoided if countries had a way of committing themselves to technology adoption in the long-run, or alternatively, if they firmly expect that the actions taken by other countries will be collectively rational responses to their own actions, even in the long-run. In practice, however, countries may be hard-pressed to find such a commitment device given

5 Note that a country with the conventional technology abates $q=\frac{b}{c_{0}}$. For the frontrunners' payoff to adoption we use that the payoff is given by: $\Pi_{i}=b_{x} M-\frac{c_{x}}{N}(N-M+1)+\beta\left[b_{x} N-\frac{c_{x}}{N}\right]$ if in period 2 all followers unanimously adopt Technology $X$, and by:

$$
\Pi_{i}=b_{x} M-\frac{c_{x}}{N}(N-M+1)+\beta\left[b_{x} M-\frac{c_{x}}{N}(N-M+1)+\frac{b^{2}}{2 c_{0}}(N-M)\right]
$$

if no follower adopts Technology X. Finally, payoffs are given by $\Pi_{i}=\frac{b^{2}}{2 c_{0}}(M-1)+\beta\left[\frac{b^{2}}{2 c_{0}}(N-1)\right]$ if no country adopts $X$ throughout the two periods. 
that technology implementation covers a long time span and that political decision-makers face uncertainty about future election outcomes, economic growth, and the pace of technological progress. Perhaps as a reflection of this fact, the Kyoto Protocol had a commitment period of only five years.

The simple model discussed in this section shows that the outcome of a technologyoriented treaty is partly determined by members' subjective beliefs about future technology adoption, or, more precisely, about the collective capacity to coordinate technology adoption in the future. Countries' perceptions might be influenced by visible commitments to

(a)

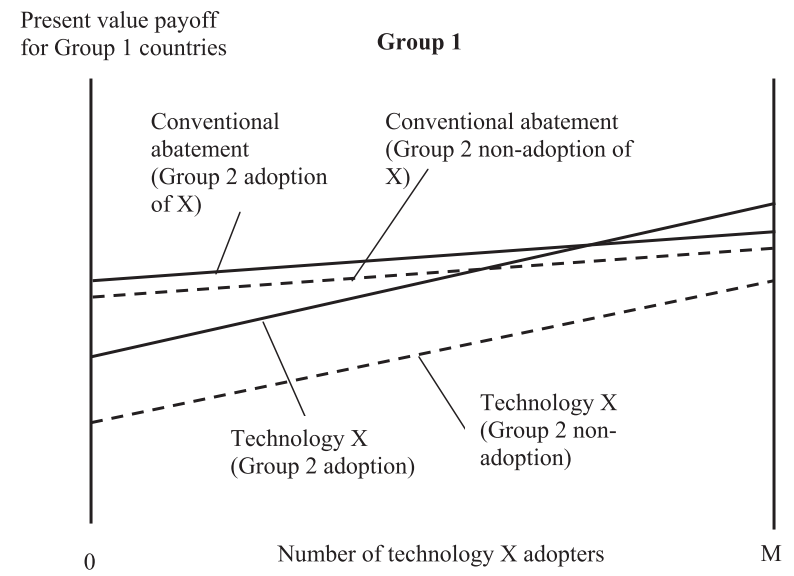

Fig. 1a. Adoption incentives under irreversibililty: payoff to frontrunners.

(b)

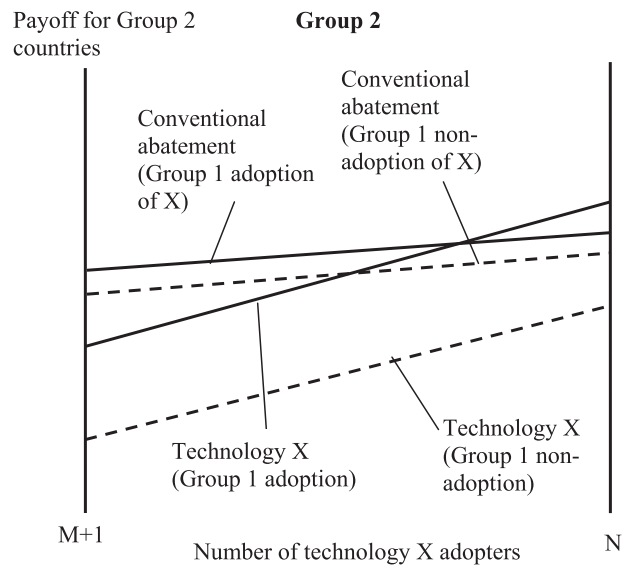

Fig. 1b. Adoption incentives under irreversibility: payoff to followers. 
solving an international environmental problem, in addition to adopting the breakthrough technology, such as the voluntary adoption of a long-term emission target.

\section{Indeterminate technology diffusion when countries choose the time of adoption}

This section extends the baseline model by dropping the assumptions of irreversibility and of a fundamental asymmetry between frontrunners and followers. We rather allow for technology switching in both ways, i.e., both adoption and abandonment of Technology $\mathrm{X}$ are possible at a cost. We show that the two-stage game can have multiple equilibria as in the previous section, and that the solutions to the infinite-horizon game resemble those of the two-period case. We also discuss the equilibrium dynamics of the infinite-horizon case.

\subsection{Two-period case}

We retain the assumption of two periods but now assume that $M_{0}$ countries $\left(0 \leq M_{0} \leq N\right)$ have adopted Technology $\mathrm{X}$ already before period 1 , and that $\Delta M_{1}$ countries seek the introduction of Technology $\mathrm{X}$ in period 1 . Note that here we use a general formulation of $M_{0}$ that does not require $M_{0}$ to be zero (although it can be). In so doing, we account for the possibility that Technology $\mathrm{X}$ has been adopted by some countries because of existing or past treaties, or for reasons unrelated to climate change. By introducing an assumption of switching cost, which is to be described below, we eliminate the distinction between frontrunners and followers used in Section 2. That is, the cost structure and technological capacity are assumed to be identical for all countries, and all countries can adopt Technology $\mathrm{X}$ from period 1 .

In addition to the model primitives described in the previous section, we introduce a term $S C$ representing the marginal costs of switching the technology. We assume that $S C$ increases with the number of countries switching in the same time period. This assumption is motivated by the observation that adopting a new technology often requires the installation of new physical capital. If the industries that provide the new technology and installation services operate with decreasing returns to scale (e.g., because some production factors are fixed), the resulting supply curve of installation services is upward sloping, and hence marginal costs are increasing with the number of entities switching at a given time. ${ }^{6}$ A similar logic is used by Mussa (1978) and Krugman (1991) for their modelling of cross-industry switching costs.

We adopt this formulation to our case and assume that a switching country incurs costs $S C=f \Delta M_{1}$, where $f$ is a positive constant and $\Delta M_{1}$ is the number of countries adopting Technology X. Note that $S C$ concerns pure adjustment costs for the adopting countries and is independent of the existing adopters of the technology-hence, it is not a function of $M_{0}$

6 For example, convex costs are likely to arise when countries switch from nuclear energy to other forms of carbon-neutral electricity generation, as was decided by the German government after the Fukushima nuclear accident ('Energiewende'). Since Germany is the only country that has taken this step so far, it will be able to import cheap nuclear power from other European countries during the transition to a nuclear free electricity supply. However, if other European countries adopt similar decisions, this is bound to drive up the initial cost of technology switching as countries would bid up the price of (nuclear) power. Moreover, unchecked growth in transnational electricity trade could lead to congestion on the European transmission grid. 
but only of $\Delta M_{1}$. We assume that $f>\frac{c_{x}}{N}(1+\beta)$, i.e., the switching cost outweighs the (present-value) externality effect on running costs for Technology X. For the sake of simplicity, we assume that a symmetrical moving cost is incurred when $-\Delta M_{1}$ countries abandon Technology $\mathrm{X}$ and switch to the conventional abatement.

Countries decide upon adoption taking into account the one-time switching cost as well as the present-value gain associated with using Technology $\mathrm{X}$ instead of the conventional abatement option. Let $\lambda$ denote this gain. Note that individual countries make decisions according to their own expected payoffs, and thus $\lambda$ represents the gain for individual countries, not for all countries. $\lambda$ is a function of $M_{0}, \Delta M_{1}$, and $\Delta M_{2}$ (the number of countries that switch technologies in period 2) given by:

$$
\lambda\left(M_{0}, \Delta M_{1}, \Delta M_{2}\right)=(1+\beta) \lambda^{1}\left(M_{0}\right)+\lambda^{2}\left(\Delta M_{1}, \Delta M_{2}\right) .
$$

where $\lambda^{1}$ and $\lambda^{2}$ are defined as:

$$
\begin{aligned}
& \lambda^{1}\left(M_{0}\right)=b_{x}-\frac{c_{x}}{N}\left(N-M_{0}+1\right)-\frac{b^{2}}{2 c_{0}} \\
& \lambda^{2}\left(\Delta M_{1}, \Delta M_{2}\right)=(1+\beta) \Delta M_{1} \frac{c_{x}}{N}+\beta \Delta M_{2} \frac{c_{x}}{N}
\end{aligned}
$$

The balance of $\lambda$ and the switching cost determines the number of adoption or abandonment of Technology $\mathrm{X}$ in period 1. Let us first consider the case of progressive technology adoption, i.e., the number of adopters of Technology $\mathrm{X}$ increases over time. Then there is a maximum value of $\Delta M_{1}$ (less than $N-M_{0}$ ) such that the payoff gain from switching from conventional abatement to Technology $\mathrm{X}$ is positive (recall that a larger $\Delta M_{1}$ reduces the expected payoff of adoption because of the switching cost). In equilibrium, the number of countries switching technologies in period $1, \Delta M_{1}^{e}$, is given by the largest integer to satisfy:

$$
\lambda\left(M_{0}, \Delta M_{1}, \Delta M_{2}\right) \geq f \Delta M_{1}
$$

In other words:

$$
\lambda\left(M_{0}, \Delta M_{1}^{e}, \Delta M_{2}\right) \geq f \Delta M_{1}^{e}
$$

and:

$$
\lambda\left(M_{0}, \Delta M_{1}^{e}+1, \Delta M_{2}\right)<f \cdot\left(\Delta M_{1}^{e}+1\right) .
$$

By contrast, countries might expect that others will abandon Technology X in period 2. As Technology $\mathrm{X}$ is attractive only with a large number of adopters, the fear of collective abandonment gives those that have adopted Technology $\mathrm{X}$ an incentive to abandon it. As above, switching costs limit the magnitude of abandonment in this period. For a set of negative $\Delta M_{1}$ that satisfy:

$$
-\lambda\left(M_{0}, \Delta M_{1}, \Delta M_{2}\right) \geq-f \Delta M_{1}
$$

the number of countries that abandon Technology $\mathrm{X}$ in period $1, \Delta M_{1}^{e}$, is given by the integer with the largest absolute value in this set.

It is straightforward to show possible cases in which the outcome is indeterminate, so that expectations about future outcomes can influence the future outcomes themselves. Figure 2 depicts the case where $M_{0}$ is located to the left of the tipping point 
(a)

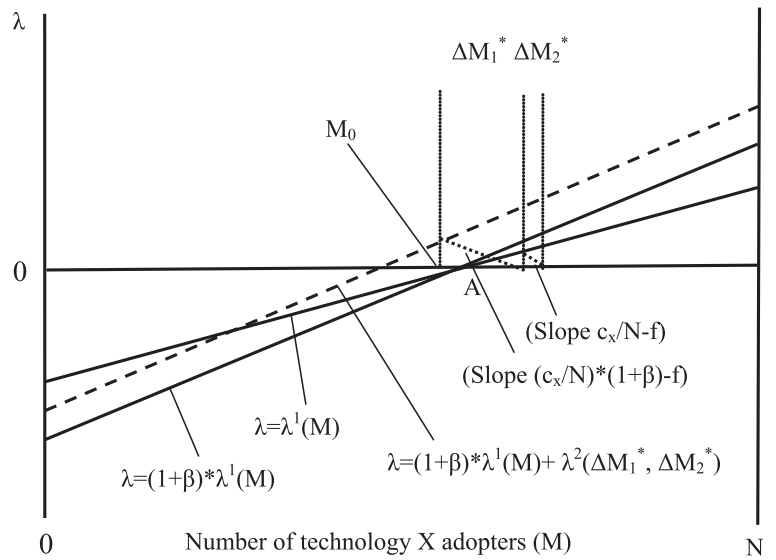

Fig. 2a. Adoption incentives with costly technology switching: technology adoption.

(b)

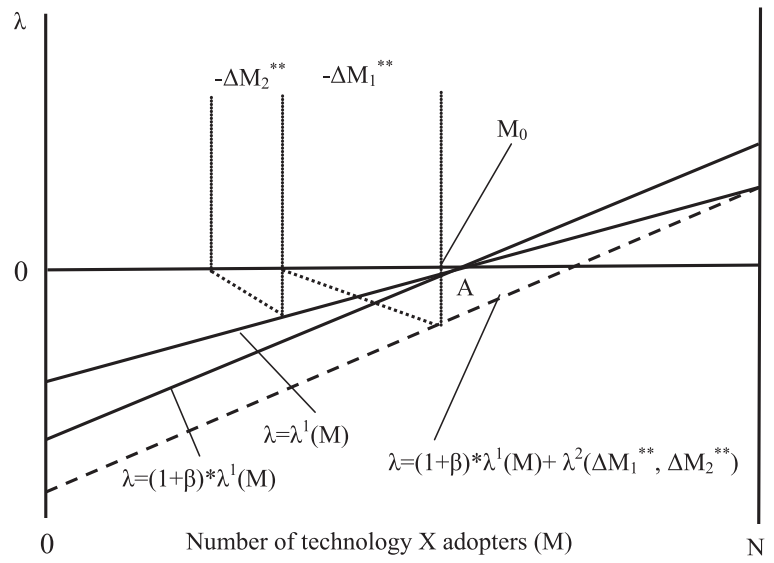

Fig. 2b. Adoption incentives with costly technology switching: technology abandonment.

A-mathematically, $\lambda^{1}\left(M_{0}\right)=0$. We prove in Appendix A.1 that there is always at least one combination of $\left(\Delta M_{1}, \Delta M_{2}\right)$ satisfying (11) and $\Delta M_{1}, \Delta M_{2} \leq 0$ (Fig. 2b). Under certain conditions, there may also be a combination of $\left(\Delta M_{1}, \Delta M_{2}\right)$ satisfying (8) and $\Delta M_{1}, \Delta M_{2} \geq$ 0 (Fig. 2a). To see this, notice that-by a logic similar to the one used to derive condition (8) - the number of countries that adopt Technology $\mathrm{X}$ in period $2, \Delta M_{2}^{e}$, is given by the largest integer of $\Delta M_{2}$ to satisfy the inequality:

$$
\lambda^{1}\left(M_{0}+\Delta M_{1}+\Delta M_{2}\right) \geq f \Delta M_{2}
$$


The above conditions (8) and (12) are equivalent to:

$$
\begin{gathered}
\frac{N}{c_{x}} \lambda^{1}\left(M_{0}\right)+\left(1-\frac{f N}{c_{x}(1+\beta)}\right) \Delta M_{1}+\frac{\beta}{1-\beta} \Delta M_{2} \geq 0 \\
\frac{N}{c_{x}} \lambda^{1}\left(M_{0}\right)+\Delta M_{1}+\left(1-\frac{f N}{c_{x}}\right) \Delta M_{2} \geq 0
\end{gathered}
$$

and can be satisfied by a set of weakly positive $\left(\Delta M_{1}, \Delta M_{2}\right)$. For example, positive $\Delta M_{1}$ and $\Delta M_{2}$ exist if $\frac{f N}{c_{x}(1+\beta)}-1$ is very small (recall that, by assumption, $\frac{f N}{c_{x}(1+\beta)}-1>0$ ) and there is a number $\Delta M_{2}$ that satisfies:

$$
\frac{\beta}{1-\beta} \Delta M_{2}>-\frac{N \lambda^{1}\left(M_{0}\right)}{c_{x}}
$$

(note that $\lambda^{1}\left(M_{0}\right)<0$ ). An analogous reasoning can be developed for the case in which $M_{0}$ is located to the right of the tipping point.

In summary, we have shown that the dynamics of technology switching may be uniquely determined in the direction of either increasing adoption or abandonment, depending on the initial state of technology adoption. However, the system may also have feasible solutions for both directions of technology adoption and abandonment, in which case the outcome is determined entirely by countries' expectations.

\subsection{Infinite-horizon game}

Here we show that similar patterns to the ones described in the previous section emerge in the case of an infinite-horizon game of technology adoption. In this setting, the effect of actions at any given stage is cumulative so that final outcomes differ drastically, depending on both the model primitives and players' expectations. There are two fundamentally different scenarios. In the first one, the dynamics are determinate, in the sense that equilibrium play always leads to a unique outcome, either a universal adoption or zero adoption. In the second scenario, the dynamics are indeterminate, so that expectations about future outcomes influence the future outcomes themselves, akin to a self-fulfilling prophecy.

We analyse an infinite-horizon version of the game developed in the previous section. Play starts in period 0 with an initial number $M_{0}$ of adopters. As in the previous section, we assume that $0 \leq M_{0} \leq N$ to allow for the possibility that some countries have already adopted Technology X. Countries maximize the present value of cumulative expected future payoffs associated with their chosen technology. We focus on subgame perfect equilibria with the feature that countries immediately begin an optimal transition to either full adoption or no adoption-which one depends on $M_{0}$, payoff parameters, and expectations. Once this stage-game Nash equilibrium is reached, it will be repeated indefinitely as players have no incentives to further deviate. Since indefinite Nash play is a subgame perfect equilibrium of the continuation game, we can use backward induction to determine the individually rational transition towards this state. In the following exposition, we first consider the case in which all countries eventually become adopters of Technology X. An analogous reasoning can be made for technology abandonment, as shown below.

Due to switching costs, universal adoption does not occur in a single period but will take place in $L$ batches $\Delta M_{1}, \Delta M_{2}, \ldots, \Delta M_{L-1}, \Delta M_{L}$ where $\sum_{l=1}^{L} \Delta M_{l}=N-M_{0}$. Consider the last batch of $\Delta M_{L}=N-M_{L-1}$ of adopters. The relative payoff to adoption for these countries is given by: 


$$
\lambda^{L}=\frac{1}{1-\beta}\left[b_{x}-\frac{c_{x}}{N}\left(N-M_{0}+1\right)-\frac{b^{2}}{2 c_{0}}+\frac{c_{x}}{N}\left(\sum_{l=1}^{L} \Delta M_{l}\right)\right]
$$

A Nash equilibrium in this subgame requires that $\lambda^{L} \geq f \Delta M_{L}$ where $\Delta M_{L}=N-M_{L-1}$. Working backwards, in period $L-1$ a group of $\Delta M_{L-1}$ countries adopting Technology X earns relative payoffs:

$$
\lambda^{L-1}=\frac{1}{1-\beta}\left[b_{x}-\frac{c_{x}}{N}\left(N-M_{0}+1\right)-\frac{b^{2}}{2 c_{0}}+\frac{c_{x}}{N}\left(\sum_{l=1}^{L-2} \Delta M_{l}+\Delta M_{L-1}+\beta \Delta M_{L}\right)\right]
$$

For there to be exactly $\Delta M_{L-1}$ adopters in Nash equilibrium, adoption must make all of them weakly better off, i.e.:

$$
\lambda^{L-1} \geq f \Delta M_{L-1} .
$$

However, any additional adopter of Technology X must be strictly worse off:

$$
\lambda^{L-1}+\frac{c_{x}}{N}<f\left(\Delta M_{L-1}+1\right)
$$

Iterating backwards, we obtain the relative payoff to adoption on the equilibrium path for the $k$ th batch of adopters, $k \in\{1, L-1\}$ :

$$
\lambda^{k}=\frac{1}{1-\beta}\left[b_{x}-\frac{c_{x}}{N}\left(N-M_{0}+1\right)-\frac{b^{2}}{2 c_{0}}+\frac{c_{x}}{N}\left(\sum_{l=1}^{k-1} \Delta M_{l}+\sum_{l=k}^{L} \beta^{l-k} \Delta M_{l}\right)\right]
$$

and the equilibrium conditions:

$$
\begin{aligned}
\lambda^{k} & \geq f \Delta M_{k} \\
\wedge \lambda^{k} & <f \Delta M_{k}+f-\frac{c_{x}}{N}
\end{aligned}
$$

For given $\lambda^{k}$, conditions (20) and (21) pin down the number of adopters in a Nash equilibrium at stage $k$. Once all countries have adopted in period $L$, the relative payoff to adoption in all subsequent periods $\tau$ is constant and given by $\lambda^{L+\tau}=\frac{1}{1-\beta}\left[b_{x}-\frac{c_{x}}{N}-\frac{b^{2}}{2 c_{0}}\right]$ for $\tau=0,1, \ldots$. Since this term is positive, no country has an incentive to unilaterally abandon Technology X.

We use this property to characterize the evolution of the relative payoff to adoption along the equilibrium path. The relative payoff to adoption for countries in the $k$ th batch of adopters is given by:

$$
\lambda^{k}=\sum_{s=k}^{L} \beta^{s-k}\left[b_{x}-\frac{c_{x}}{N}\left(N-M_{s}+1\right)-\frac{b^{2}}{2 c_{0}}\right]+\frac{\beta^{L-k+1}}{1-\beta}\left[b_{x}-\frac{c_{x}}{N}-\frac{b^{2}}{2 c_{0}}\right]
$$

The difference in the relative payoffs to adoption for two subsequent batches of adopters $k$ and $k+1$ can be written as:

$$
\lambda^{k+1}-\lambda^{k}=\delta \lambda^{k}-(1+\delta)\left[b_{x}-\frac{c_{x}}{N}\left(N-M_{k}+1\right)-\frac{b^{2}}{2 c_{0}}\right]
$$

where $\delta \equiv \frac{1-\beta}{\beta}$. See Appendix A.2 for a derivation of this equation. Along with the inequalities (20) and (21), eq. (23) characterizes the dynamics of technology adoption in subgame 
perfect equilibrium. As in Section 3.1 above, the conditions for an equilibrium in which all countries switch back to the conventional technology can be derived in an analogous fashion.

\subsection{Equilibrium dynamics}

To analyse the dynamics of technology adoption along the equilibrium path, it is convenient to model the rate of technology adoption as a continuous variable $\gamma(0 \leq \gamma \leq 1)$. The number of countries adopting Technology $\mathrm{X}$ is thus given by $\lceil\gamma N\rceil$. Similar to the case discussed in the previous section, the equilibrium level of countries switching at each time period is one that balances the net present value of switching and the marginal switching costs for all countries. Along the equilibrium path, the net present value of switching from the conventional abatement to Technology $\mathrm{X}$ at period $t$ is given by:

$$
\lambda_{t}=\sum_{s=t}^{\infty} \beta^{s-t}\left[b_{x}-c_{x}\left(1-\gamma_{s}\right)-\frac{b^{2}}{2 c_{0}}\right] .
$$

Following the same logic as in the previous sub-sections, the marginal switching cost for countries switching technologies between $t$ and $t+1$ is proportional to $\left(\gamma_{t+1}-\gamma_{t}\right)$ and defined as $F\left(\gamma_{t+1}-\gamma_{t}\right)$ where $F$ is a constant. For $\lambda_{t}$ continuous in $\gamma$, conditions (20) and (21) boil down to the difference equation:

$$
F\left(\gamma_{t+1}-\gamma_{t}\right)=\frac{1}{1+\delta} \lambda_{t+1}
$$

A second difference equation (derived in Appendix A.3) governs the evolution of $\lambda_{t}$ :

$$
\lambda_{t+1}-\lambda_{t}=\delta \lambda_{t}-(1+\delta)\left[b_{x}-c_{x}\left(1-\gamma_{t}\right)-\frac{b^{2}}{2 c_{0}}\right] .
$$

As the length of a time period goes to zero, the system of difference eqs (25) and (26) can be approximated by the differential equations:

$$
\begin{gathered}
F \dot{\gamma}=\frac{1}{1+\delta} \lambda \\
\dot{\lambda}=\delta \lambda-(1+\delta)\left[b_{x}-c_{x}(1-\gamma)-\frac{b^{2}}{2 c_{0}}\right]
\end{gathered}
$$

This representation allows for a more tractable analysis of the dynamics along the equilibrium path. Note that the system describes the dynamics of technology adoption following the countries' individual payoffs. A similar discussion for the social optimum is presented in Appendix A.4. Eqs (27) and (28) define a system of linear differential equations the solution to which is given by a combination of exponential functions. If:

$$
0<-b_{x}+c_{x}+\frac{b^{2}}{2 c_{0}}<c_{x}
$$

the system has a tipping pattern, i.e., both universal adoption and zero adoption of $\mathrm{X}$ are long-run (continuation) equilibria. In this case, the paths of $\lambda$ and $\gamma$ are obtained by tracing 
(a)

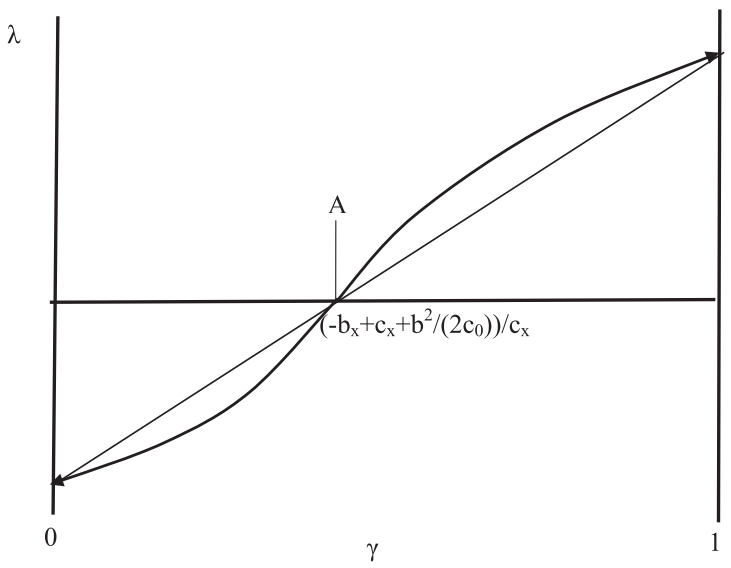

Fig. 3a. Equilibrium dynamics in the infinite horizon game: determinate case (real roots).

(b)

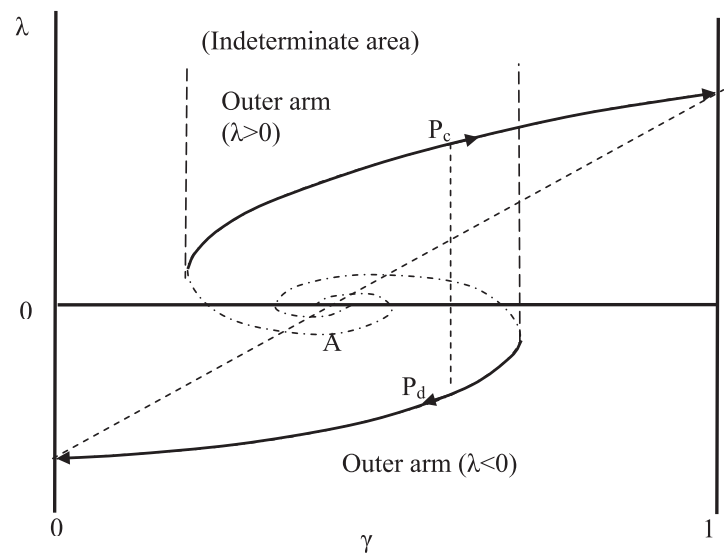

Fig. 3b. Equilibrium dynamics in the infinite horizon game: indeterminate case (complex roots).

them backwards from two long-run equilibria where $\gamma=0$ or $\gamma=1$. The roots of the exponential functions determining the system are given by:

$$
\rho=\frac{1}{2}\left[\delta \pm \sqrt{\delta^{2}-\frac{4 c_{x}}{F}}\right]
$$

Note that the roots can be both real and complex depending on the parameter values, as the term $\delta^{2}-4 c_{x} / F$ can be either positive or negative. The system dynamics exhibit remarkable differences depending on which type of root prevails in eq. (30).

With a real root, the system is determinate and hence a sequence of countries' decisions always lead to a unique outcome. This case is depicted in Fig. 3a. Starting at the tipping point A, either of the two long-run equilibria can be attained as the dynamics evolve 
through a sequence of decisions governed by the equilibrium conditions (27) and (28). The graph shows that each value of $\gamma$ other than A corresponds to at most one point on one of the two trajectories. In other words, the initial state of adoption $\gamma_{0}$ uniquely determines the long-run diffusion rate of Technology X. If $\gamma_{0}>A\left(\gamma_{0}<A\right)$ universal (zero) adoption results in long-run equilibrium. ${ }^{7}$

In contrast, expectations play a prominent role when eq. (30) has a complex root. In this case, the trajectories show oscillatory patterns, and their arms could cover a wide range of possible values for $\gamma$. When the two arms overlap over an interval of $\gamma$-as is depicted in Fig. $3 \mathrm{~b}-$ the initial state does not determine the direction of the path. In fact, there is an infinite number of feasible trajectories that the system can take. Thus, the model primitives do not condition countries to follow a unique equilibrium path. Rather, it is countries' expectations about future adoption of Technology X that pace the growth (or decline) of technology penetration. Even if there is a feasible equilibrium path leading to the universal adoption of Technology $\mathrm{X}$ (for example, the path through point $P_{c}$ in Fig. $3 \mathrm{~b}$ ), an expectation held by non-adopters that diffusion will not happen could prevent the initial group of adopters from taking this path. Instead, they might follow the trajectory to the zero adoption (for example, the path through point $P_{d}$ in Fig. 3b).

\section{Discussion}

\subsection{Patterns of technology choice}

The dynamic model highlights two distinct patterns of technology choice under a technology treaty which deserve further discussion from a policy point-of-view. In the determinate case, there is a unique equilibrium path leading to the long-run outcome. This outcome can be either universal or zero adoption and is uniquely determined by the initial state of technology adoption $\gamma_{0}$ and by the tipping point:

$$
\gamma^{*}=1-\frac{b_{x}-\frac{b^{2}}{2 c_{0}}}{c_{x}}
$$

Only if the initial proportion of adopters is sufficiently large, $\gamma^{*}<\gamma_{0}$, will the technology be adopted by everyone in the long-run. ${ }^{8}$ Otherwise, all countries will switch back to the conventional technology. The tipping point is likely to be lower the more affordable the breakthrough technology, the more expensive the conventional technology and the larger the relative benefits of Technology X compared to those associated with the conventional technology. In this scenario, the earlier results by Barrett (2006) and Hoel and de Zeeuw (2010) go through and the coordination problem is negligible.

However, this is not true if the technology is such that the long-run outcome is indeterminate and depends on expectations, akin to a self-fulfilling prophecy. Eq. (30) implies that

7 Adoption could take either of the trajectories if $\gamma_{0}=A$.

8 Conditions (30) and (31) characterize the outcomes of private decisions taken by individual countries that intertemporally maximize their cumulative payoffs. These outcomes do not necessarily coincide with the social optimum because individual countries do not consider the external benefits and costs of their switching decisions. However, similar conditions can be obtained for the social optimum, as shown in Appendix A.4. These conditions imply that the tipping point for the social optimum $\left(\hat{\gamma}^{*}\right)$ is always lower than the tipping point for private outcomes $\left(\gamma^{*}\right)$. Intuitively, this property derives from the fact that technology adoption by one country creates a positive externality for other countries adopting Technology X. 
this case arises if $\delta^{2}<4 c_{x} / F$ and hence the system of differential equations has complex roots. It is easily seen from this inequality that a higher discount rate $\delta$ and a higher switching cost $F$ parameter both promote determinacy of the system. This is because both myopia and high costs of technology switching enhance the relative importance of current over future payoffs, which are subject to strategic uncertainty. Conversely, a large $c_{x}$-which implies that the costs of technology adoption very much depend on the total number of adopters-promotes indeterminacy of the system, as it makes countries' present-value expected payoff more susceptible to others' technology choices in the future.

\subsection{Equilibrium refinements for coordination games}

The issue of coordinating play on one of several possible equilibrium outcomes is at the core of this and other papers on breakthrough technologies. In any coordination game, players face strategic uncertainty about the decisions taken by other players. Our theoretical model has shown how introducing dynamics exacerbates strategic uncertainty by creating indeterminacy. This result highlights the role of expectations that may affect future technology adoption in a self-fulfilling fashion, leading to the implication that policymakers may wish to coordinate expectations on an agreement with full adoption. An alternative approach would have been to incorporate more structure on player's expectations in the model and hence to narrow the scope for multiplicity. ${ }^{9}$ In this subsection, we discuss the available game theoretical concepts to resolve coordination issues and explain why we have refrained from using them.

Schelling (1960) pointed out early on that beliefs and perceptions held by players can help coordinate expectations on certain outcomes that are focal. This is particularly true of real-life situations where agents' decisions are embedded in a common temporal, spatial or cultural context. In the description of the technology adoption game considered above, however, there is little that would render a particular Nash equilibrium focal in Schelling's sense, so this concept is not pursued any further here.

Harsanyi and Selten (1988) defined the notion of risk dominance as an equilibrium refinement for (static) coordination games. ${ }^{10}$ This concept is nicely illustrated for the stage game depicted in Fig. 2a. If all frontrunners expect followers to adopt Technology X, they play a coordination game with payoffs represented by the solid lines. It is easily seen that complete adoption by all frontrunners payoff-dominates the Nash equilibrium with no adoption. However, notice that a deviation by one of the frontrunners imposes larger losses on the other adopters than would be the case in the equilibrium with no adoption.

9 We thank an anonymous referee for making this suggestion.

10 For anti-coordination games such as the game of chicken, Aumann (1974) proposed the concept of correlated equilibrium, where players coordinate expectations on equilibrium play via a third party that privately instructs players which strategy to play after observing to a randomization device. We refrain from using correlated equilibria here because, in line with the previous literature, we think that the choice of the abatement technology is best modelled as a coordination game rather than an anti-coordination game. Moreover, it would appear heroic to assume that an international body such as the United Nations Framework Convention on Climate Change (UNFCCC) could assign equilibrium actions to its member states in private. Rather, it seems that such assignments should be considered public in a post-'Wiki leaks' world. As a result, the UNFCCC could only randomize over Nash equilibria of the game, but this is not very plausible. 
Therefore, if frontrunners don't know for sure which Nash equilibrium is being played, adopting Technology $\mathrm{X}$ is a risky choice whereas conventional abatement is safe. In other words, the equlibrium without adoption risk-dominates the equilibrium with complete adoption. Risk dominance seems particularly plausible if pre-play communication is ineffective at coordinating expecations, as was conjectured by Aumann (1990). However, at least in two-player coordination games, lab experiments have shown that cheap talk communication is very effective at enhancing efficiency of the outcome (Charness, 2000). Moreover, a fair amount of international diplomacy can be considered cheap talk in a game theoretical sense, and yet it is the prime method of enhancing the efficiency of intergovernmental interactions. This (along with the lack of an extensive-form game definition), is the reason why we do not use risk dominance to select among equilibria.

\subsection{Social preferences}

Our model has emphasized the scale effects of breakthrough technologies which induce strategic complementarity in the adoption process. While the examples for such technologies given above are well known in the literature, it bears noting that the fundamental insights of our analysis are much broader. In fact, they equally apply to any factor capable of creating strategic complementarity in an international environmental treaty. For example, Lange and Vogt (2003) show that the introduction of equity preferences à la Bolton and Ockenfels (2000) in Barrett's (1994) model of self-enforcing environmental treaties can sustain full cooperation in settings where regular preferences cannot. This finding is relevant in our context because a large body of experimental evidence emphasizes the importance of social preferences (Fehr and Schmidt, 1999; Bolton and Ockenfels, 2000), and because concerns about fairness have been shown to matter for those actors involved in international climate negotiations (Lange et al., 2007, 2010). Wagner (2016) presents empirical evidence that concerns about reputation and fairness created strategic complementarity in the ratification process of the Montreal Protocol on Substances that Deplete the Ozone Layer.

Similar to the scale effect of the breakthrough technology, inequality aversion engenders strategic complementarity in the payoff to joining a treaty. In particular, when countries care about a fair distribution of the gains of an international environmental treaty, the dynamic ratification path can be indeterminate even in the absence of technology externalities. Given this indeterminacy, participation in the treaty could be broadened if countries believe that other countries will become adopters in the future (i.e., ratification becomes the norm), and vice versa. The only difference is that in eq. (30), the technology term $c_{x}$ will be replaced by a parameter measuring the inequality aversion. We leave a more detailed analysis of such a model as a topic for future research.

\section{Conclusion}

Although the 2015 Paris Agreement prominently features a $2{ }^{\circ} \mathrm{C}$ target for global warming, it does not stipulate binding targets on carbon emissions. Rather, member states are called upon to design and implement their own mitigation measures, cautiously referred to as 'Intended Nationally Determined Contributions (INDCs)'. According to the EU commission, these INDCs 'are not yet enough to keep global warming below $2^{\circ} \mathrm{C}$, but the 
agreement traces the way to achieving this target. ${ }^{11}$ The theoretical model developed in this paper sheds light on the conditions under which this statement holds true.

Our analysis is based on a dynamic extension of Barrett's (2006) model of treaty formation where countries choose between: (i) a conventional abatement technology that is subject to decreasing returns; and (ii) a 'breakthrough technology' that exhibits a network externality such that the benefit to adoption increases with the number of other adopters. Countries evaluate these choices taking into account the expected future benefits and an increasing (in the number of other countries) cost of switching technologies. We have shown that, under certain conditions, equilibrium technology choices are indeterminate as they depend on the future adoption rate, which is subject to strategic uncertainty. An important implication of this is that expectations about the future outcomes could themselves influence international cooperation under an IEA that mandates the adoption of a clean technology.

Our analysis highlights the potential of strategic uncertainty to hinder treaty formation and suggests two approaches by which policy can mitigate this problem. The first approach is to reduce strategic uncertainty by managing expectations. This could be implemented, for example, by setting long-term, non-binding targets for pollution emissions or other outcomes, so as to coordinate expectations across countries on the path leading to full adoption. As was pointed out above, the fact that limiting the global temperature increase to $2{ }^{\circ} \mathrm{C}$ is the key provision of both the Copenhagen Accord and the Paris Agreement can be interpreted in this way. This target does not require any country to reduce its emissions at present, but it aligns countries' expectations and thereby 'tips' a future technology treaty towards adoption.

The second approach to reducing strategic uncertainty is by choosing technologies that minimize the potential for indeterminacy of the dynamic system. Instead of choosing the most efficient breakthrough technology, policymakers might favour a technology with high switching cost as this locks the frontrunners into their decisions while also reducing strategic uncertainty of followers. This aspect of technology adoption arises only in our explicitly dynamic framework and thus constitutes an important extension of the second-best argument by which technologies with scale effects are superior to alternative treaty designs even if they come at a higher cost because they reduce the incentive to free ride (Barrett, 2006).

For the sake of clarity, we have kept the modelling and discussion deliberately simple. Our analysis can be extended to consider expectation-driven dynamics that emerge because of sources of strategic complementarities other than a network externality, e.g., inequality aversion on the part of treaty participants or trade sanctions imposed on non-signatories. What is more, our analysis could be extended to IEAs that concern both R\&D investment and technology diffusion, and this will require a proper treatment of irreversibilities. Another relevant extension would examine the scope for expectations management in an IEA involving the choice among many technologies that exhibit different cost structures. These and other extensions are left as topics for future research.

11 Cf. https://ec.europa.eu/clima/policies/international/negotiations/paris/index_en.htm, last accessed 31 October 2016. 


\section{Funding}

This work was supported by the Spanish Ministry for Economy and Competitiveness (RYC-201312492 to U.W., ECO2012-31358 to U.W.); Spanish Ministry for Science and Innovation (JCI-200904392 to U.W.); Spanish Ministry for Science and Education (SEJ2007-62908 to U.W. ).

\section{Acknowledgements}

The authors would like to thank two anonymous referees for their insightful comments and Thomas Tröger for helpful discussions.

\section{References}

Aumann, R. (1974) Subjectivity and correlation in randomized strategies, Journal of Mathematical Economics, 1, 67-96.

Aumann, R. (1990) Nash-equilibria are not self-enforcing, in J. Gabszewicz, J.-F. Richard, and L. Wolsey, (eds) Economic Decision Making: Games, Econometrics and Optimisation, North-Holland, Amsterdam.

Barrett, S. (1994) Self-enforcing international environmental agreements, Oxford Economic Papers, 46, 878-94.

Barrett, S. (2003) Environment and Statecraft, Oxford University Press, New York.

Barrett, S. (2006) Climate treaties and 'breakthrough' technologies, American Economic Review, $96,22-5$.

Bolton, G.E. and Ockenfels, A. (2000) Self-centered fairness in games with more than two players, American Economic Review, 90, 166-93.

Charness, G. (2000) Self-serving cheap talk: a test of Aumann's conjecture, Games and Economic Behavior, 33, 177-94.

Fehr, E. and Schmidt, K.M. (1999) A theory of fairness, competition and cooperation, Quarterly Journal of Economics, 114, 817-68.

Gladwell, M. (2000) The Tipping Point: How Little Things Can Make a Big Difference, Little, Brown and Company, New York.

Harsanyi, J. and Selten, R. (1988) A General Theory of Equilibrium Selection in Games, MIT Press, Cambridge, MA.

Hoel, M. and de Zeeuw, A. (2010) Can a focus on breakthrough technologies improve the performance of international environmental agreements?, Environmental and Resource Economics, 47, 395-406.

Krugman, P. (1991) History versus expectations, Quarterly Journal of Economics, 106, 651-67.

Lange, A., Löschel, A., Vogt, C., and Ziegler, A. (2010) On the self-interested use of equity in international climate negotiations, European Economic Review, 54, 359-75.

Lange, A. and Vogt, C. (2003) Cooperation in international environmental negotiations due to a preference for equity, Journal of Public Economics, 87, 2049-67.

Lange, A., Vogt, C., and Ziegler, A. (2007) On the importance of equity in international climate policy: an empirical analysis, Energy Economics, 29, 545-62.

Mussa, M. (1978) Dynamic adjustment in the Heckscher-Ohlin-Samuelson model, Journal of Political Economy, 86, 775-91.

Parson, E.A. (2003) Protecting the Ozone Layer. Science and Strategy, Oxford University Press, Oxford.

Schelling, T.C. (1960) The Strategy of Conflict, Harvard University Press, Cambridge, MA.

Schelling, T.C. (1978) Micromotives and Macrobehavior, W.W. Norton \& Co., New York.

Sunstein, C. (2007) Of Montreal and Kyoto: a tale of two protocols, Harvard Environmental Law Review, 31, 1-66.

Wagner, U.J. (2016) Estimating strategic models of international treaty formation, Review of Economic Studies, 83, 1741-78. 


\section{Appendix}

\section{A. 1 Proof}

The given conditions imply $-\lambda\left(M_{0}, 0,0\right) \dot{\imath} 0$ and. $-\lambda^{1}\left(M_{0}\right)>0$. Since: $f>\frac{c_{x}}{N}(1+\beta)$, the function:

$$
-\lambda\left(M_{0}, \Delta M_{1}, \Delta M_{2}\right)-\lambda^{1}\left(-\Delta M_{1}\right)
$$

is increasing in $\Delta M_{1}$ (decreasing in $-\Delta M_{1}$ ) and decreasing in $\Delta M_{2}$ (increasing in $-\Delta M_{2}$ ). As, this means that there is at least one feasible $\Delta M_{1} \leq 0$ for all $\Delta M_{2}$ satisfying $\Delta M_{2} \leq 0$.

Meanwhile, a negative $\Delta M_{2}$ satisfies the following inequality:

$$
-\lambda^{1}\left(M_{0}+\Delta M_{1}+\Delta M_{2}\right) \geq f\left(-\Delta M_{2}\right)
$$

Since $f>\frac{c_{x}}{N}(1+\beta)$, the function:

$$
-\lambda^{1}\left(M_{0}+\Delta M_{1}+\Delta M_{2}\right)-f\left(-\Delta M_{2}\right)
$$

is increasing in $\Delta M_{2}$ (decreasing in $\left.-\Delta M_{2}\right)$. As $-\lambda^{1}\left(M_{1}\right)>0$, this means that there is at least one $\Delta M_{2} \leq 0$ that satisfies the above inequality for all $\Delta M_{1}$ such that $\Delta M_{1} \leq 0$. The above means that if $M_{0}$ is located on the left of the tipping point, there is always a feasible combination of $\left(\Delta M_{1}, \Delta M_{2}\right)$ such that $\Delta M_{1}, \Delta M_{2} \leq 0$.

\section{A.2 Difference equation for $\lambda$ in the discrete game}

To characterize the evolution of the relative payoff to adoption, we rewrite the relative payoff to adoption for adopters in the $k$ th batch of adopters as follows:

$$
\lambda^{k}=\sum_{s=k}^{L} \beta^{s-k}\left[b_{x}-\frac{c_{x}}{N}\left(N-M_{s}+1\right)-\frac{b^{2}}{2 c_{0}}\right]+\frac{\beta^{L-k+1}}{1-\beta}\left[b_{x}-\frac{c_{x}}{N}-\frac{b^{2}}{2 c_{0}}\right]
$$

The relative payoff to adoption for the subsequent batch of adopters $k+1$ is given by:

$$
\lambda^{k+1}=\sum_{s=k+1}^{L} \beta^{s-(k+1)}\left[b_{x}-\frac{c_{x}}{N}\left(N-M_{s}+1\right)-\frac{b^{2}}{2 c_{0}}\right]+\frac{\beta^{L-k}}{1-\beta}\left[b_{x}-\frac{c_{x}}{N}-\frac{b^{2}}{2 c_{0}}\right]
$$

Let $\delta \equiv \frac{1-\beta}{\beta}$ and calculate:

$$
\begin{aligned}
\lambda^{k+1}-(1+\delta) \lambda^{k} & =\lambda^{k+1}-\frac{\lambda^{k}}{\beta} \\
& =\sum_{s=k+1}^{L} \beta^{s-(k+1)}\left[b_{x}-\frac{c_{x}}{N}\left(N-M_{s}+1\right)-\frac{b^{2}}{2 c_{0}}\right] \\
& -\sum_{s=k}^{L} \beta^{s-k-1}\left[b_{x}-\frac{c_{x}}{N}\left(N-M_{s}+1\right)-\frac{b^{2}}{2 c_{0}}\right] \\
& =-\frac{1}{\beta}\left[b_{x}-\frac{c_{x}}{N}\left(N-M_{k}+1\right)-\frac{b^{2}}{2 c_{0}}\right]
\end{aligned}
$$

Simple manipulation of this expression yields:

$$
\lambda^{k+1}-\lambda^{k}=\delta \lambda^{k}-(1+\delta)\left[b_{x}-\frac{c_{x}}{N}\left(N-M_{k}+1\right)-\frac{b^{2}}{2 c_{0}}\right]
$$


A.3 Difference equation for $\lambda$ with a continuum of countries

$$
\lambda_{t}=\sum_{s=t}^{\infty} \beta^{s-t}\left[b_{x}-c_{x}\left(1-\gamma_{s}\right)-\frac{b^{2}}{2 c_{0}}\right]
$$

and

$$
\lambda_{t+1}=\sum_{s=t+1}^{\infty} \beta^{s-t-1}\left[b_{x}-c_{x}\left(1-\gamma_{s}\right)-\frac{b^{2}}{2 c_{0}}\right]
$$

Calculate:

$$
\begin{aligned}
\left(\lambda_{t+1}-\lambda_{t}\right)-\delta \lambda_{t} & =\sum_{s=t+1}^{\infty} \beta^{s-(t+1)}\left[b_{x}-c_{x}\left(1-\gamma_{s}\right)-\frac{b^{2}}{2 c_{0}}\right] \\
& -\underbrace{(1+\delta)}_{=1 / \beta} \sum_{s=t}^{\infty} \beta^{s-t}\left[b_{x}-c_{x}\left(1-\gamma_{s}\right)-\frac{b^{2}}{2 c_{0}}\right] \\
& =\sum_{s=t+1}^{\infty} \beta^{s-(t+1)}\left[b_{x}-c_{x}\left(1-\gamma_{s}\right)-\frac{b^{2}}{2 c_{0}}\right] \\
& -\sum_{s=t}^{\infty} \beta^{s-t-1}\left[b_{x}-c_{x}\left(1-\gamma_{s}\right)-\frac{b^{2}}{2 c_{0}}\right] \\
& =-\frac{1}{\beta}\left[b_{x}-c_{x}\left(1-\gamma_{t}\right)-\frac{b^{2}}{2 c_{0}}\right]
\end{aligned}
$$

Hence:

$$
\begin{aligned}
& \lambda_{t+1}-\lambda_{t}=\delta \lambda_{t}-\frac{1}{\beta}\left[b_{x}-c_{x}\left(1-\gamma_{t}\right)-\frac{b^{2}}{2 c_{0}}\right] \\
& =\delta \lambda_{t}-(1+\delta)\left[b_{x}-c_{x}\left(1-\gamma_{t}\right)-\frac{b^{2}}{2 c_{0}}\right]
\end{aligned}
$$

\section{A.4 Social optimum (in the continuous-time case)}

Taking the perspective of the global social planner, the Hamiltonian of the system is given by:

$$
H=N \gamma\left[b_{x}-c_{x}(1-\gamma)\right]+N(1-\gamma) \frac{b^{2}}{2 c_{0}}-\frac{N F \dot{\gamma}^{2}}{2}+N \hat{\lambda} \dot{\gamma}
$$

where $\hat{\lambda}$ is the co-state variable representing the shadow value of having an adopter of Technology $\mathrm{X}$ rather than a non-adopter. The control and state variables for the Hamiltonian are $\dot{\gamma}$ and $\gamma$. The first-order conditions are:

$$
\begin{aligned}
& \frac{\partial H}{\partial \dot{\gamma}}=-N F \dot{\gamma}+N \hat{\lambda}=0 \\
& \frac{d \hat{\lambda}}{d t}=\dot{\hat{\lambda}}=\delta \hat{\lambda}-\left[2 c_{x} \gamma+b_{x}-c_{x}-\frac{b^{2}}{2 c_{0}}\right]
\end{aligned}
$$


Just as in the case of individual countries' decision-making, this set of equations is solvable, and now the roots are given by:

$$
\hat{\rho}=\frac{1}{2}\left[\delta \pm \sqrt{\delta^{2}-\frac{8 c_{x}}{F}}\right]
$$

The tipping point now becomes:

$$
\hat{\gamma}^{*}=\frac{1}{2}\left(1-\frac{b_{x}-\frac{b^{2}}{2 c_{0}}}{c_{x}}\right)
$$

Note that $\gamma^{*}>\hat{\gamma}^{*}$. 